\title{
Layered Clay-Alginate Composites for the Adsorption of Anionic Dyes: A Biocompatible Solution for Water/Wastewater Treatment
}

\author{
Sanil Sebastian ${ }^{1,2}$, S. Mayadevi ${ }^{1^{*}}$, B. Sajeena Beevi ${ }^{2}$, Sujata Mandal ${ }^{3}$ \\ ${ }^{1}$ Chemical Engineering and Process Development Division, CSIR-National Chemical Laboratory, Pune, India \\ ${ }^{2}$ Chemical Engineering Department, Government Engineering College, Trissur, India \\ ${ }^{3}$ Expertise Center for Eco-Testing Laboratory (EXCEL), CSIR-Central Leather Research Institute, Chennai, India \\ Email: sebastian.sanil@gmail.com, s.mayadevi@ncl.res.in, sajeenanazer@gmail.com, sujata@clri.res.in
}

Received December 26, 2013; revised January 21, 2014; accepted February 18, 2014

Copyright (C) 2014 Sanil Sebastian et al. This is an open access article distributed under the Creative Commons Attribution License, which permits unrestricted use, distribution, and reproduction in any medium, provided the original work is properly cited. In accordance of the Creative Commons Attribution License all Copyrights (C) 2014 are reserved for SCIRP and the owner of the intellectual property Sanil Sebastian et al. All Copyright (C) 2014 are guarded by law and by SCIRP as a guardian.

\begin{abstract}
Adsorption properties of layered anionic clay-composites for the removal of anionic dyes from water were studied. The dye adsorption capacities of the composites were higher than the pristine clay. The clay-alginate composite with $5.9 \%$ alginate showed highest adsorption for both the dyes. The maximum adsorption capacity of the composite was enhanced by $51 \%$ for Acid Green 25 and $160 \%$ for Acid Green 27, compared to the pristine layered clay sample. The adsorption isotherm data were fitted to the Langmuir, Freundlich and Temkin isotherm models. The isotherm data could be explained well using the Freundlich isotherm model. Adsorption kinetics was analyzed using normal first order and Lagergren first order kinetic models.
\end{abstract}

\section{KEYWORDS}

Layered Clay; Sodium Alginate; Composite; Adsorption; Acid Green 25; Acid Green 27; Isotherm; Kinetics

\section{Introduction}

Clay-polymer composites and nanocomposites have gained increasing attention worldwide academically as well as industrially due to the superior properties they exhibit compared to either the clay or the polymer. Besides, they have the advantage that their properties can be tailored according to the requirement. A wide variety of composites/nanocomposites with different clay-polymer combinations have been synthesized and used for various applications [1]. Application of different clay-polymer composites and nanocomposites for water treatment is well documented [2-5]. A large number of the studies report use of clay minerals like, montmorillonite, Bentonite etc., with different polymers for the syntheses of claypolymer nanocomposites for water treatment applications [4-8]. However, limited studies report the use of layered anionic clays for making clay-polymer composites for

${ }^{*}$ Corresponding author. removing various toxic materials from water $[9,10]$.

Layered anionic clays, also known as layered double hydroxides (LDHs), have been found to be good adsorbents for ground water/waste water treatment [11-13]. Synthesis of biocompatible composites and their application in water treatment has been a recent research area of increasing interest. Alginate is a naturally occurring carbohydrate polymer often used for immobilization of biological entities [14]. It can offer biocompatibility to synthetic anionic clays by composite formation. We have recently reported the synthesis of alginate and layered anionic clay composites and their application in fluoride and Orange II dye removal [15]. These composites exhibited enhanced adsorption capacity for the adsorption of fluoride and Orange II dye from aqueous solution.

The present study reports the synthesis of layered clayalginate composites with varying amount of sodium alginate (MgAl-Alg, wt\%: 3, 5.9, 11 and 20). Adsorption characteristics of the synthesized composites (MgAl-Alg) 
are studied for the adsorption of anthraquinone dyes, Acid Green 25 (AG 25) and Acid Green 27 (AG 27) for the first time. The dye adsorption capacity of the pristine clay (MgAl) increased considerably on formation of composite with sodium alginate (Alg) and was dependent on the alginate concentration of the composite. A detailed study on the influence of alginate concentration on the dye adsorption capacity, adsorption isotherms and kinetics of adsorption is presented in this paper. Being a biopolymer, sodium alginate is expected to offer biocompatibility to the synthesized clay-alginate composite adsorbents.

\section{Materials and Methods}

\subsection{Materials}

Layered anionic clay (MgAl) was synthesized from anhydrous $\mathrm{AlCl}_{3}$, and $\mathrm{MgCl}_{2} \cdot 6 \mathrm{H}_{2} \mathrm{O}$ (AR Grade, sd Fine Chemicals Ltd., India). AR grade $\mathrm{NaOH}$ pellets were used for co-precipitation (MERCK Chemicals). Sodium alginate was procured from HiMedia Laboratory Chemicals and Biochemicals Pvt. Ltd., India. Double distilled water was used for the syntheses. The dyes AG 25 and AG 27 used in the adsorption studies were procured from Aldrich Chemicals.

\subsection{Synthesis of Adsorbents}

Layered anionic clay (MgAl) and its composites with varying concentrations of sodium alginate (Alg) were prepared by co-precipitation following the methods described in our previous articles [11,15]. MgAl was prepared by co-precipitation in an aqueous solution containing anhydrous $\mathrm{AlCl}_{3}$ and $\mathrm{MgCl}_{2} \cdot 6 \mathrm{H}_{2} \mathrm{O}$ (Mg: $\mathrm{Al}$ molar ratio of 2) by $2 \mathrm{M} \mathrm{NaOH}$ solution at $60^{\circ} \mathrm{C}$ and a $\mathrm{pH}$ of 10 \pm 0.5 . The precipitate formed was aged for $24 \mathrm{~h}$ under continuous stirring, separated using centrifuge and repeatedly washed with distilled water till the washing was neutral to litmus and subsequently dried at $60^{\circ} \mathrm{C}$ in an air oven till constant weight was obtained.

MgAl-Alg composites with different alginate concentrations ( $3 \mathrm{wt} \%, 5.9 \mathrm{wt} \%, 11 \mathrm{wt} \%$ and $20 \mathrm{wt} \%$ ) were synthesized by in-situ co-precipitation. A fine suspension of sodium alginate was made by adding required weight of sodium alginate in $50 \mathrm{ml}$ of distilled water at $60^{\circ} \mathrm{C}$. Co-precipitation of the clay was performed in the sodium alginate-water suspension (Mg:Al molar ratio of 2) using $2 \mathrm{M} \mathrm{NaOH}$ solution following the above mentioned procedure for the synthesis of MgAl. Five different adsorbents, MgAl, MgAl-Alg (3\%), MgAl-Alg (5.9\%), MgAlAlg (11\%) and MgAl-Alg (20\%) were synthesized and used in this study.

\subsection{Characterization Techniques}

The mineralogical phases and crystallinity of $\mathrm{MgAl}$ and its composites with sodium alginate were studied using X-ray diffraction (XPERT-PRO XRD from PANalytical Instruments using $\mathrm{CuK}_{\alpha}$ radiation). The morphology of the adsorbent surface was obtained by scanning electron microscopy (Leica Stereoscan 440 Scanning Electron Microscope). The specific surface area of MgAl-Alg composites, pristine $\mathrm{MgAl}$ and sodium alginate was determined by Autosorb 1C from Quantachrome Instruments, USA.

\subsection{Batch Adsorption Experiments}

Batch adsorption experiments at room temperature $\left(25^{\circ} \mathrm{C}\right)$ were performed in a thermostatic shaker (Julabo SW-21C) to study the adsorption characteristics of the clay and its composites for the dyes AG 25 and AG 27. Isotherm data was collected by contacting $100 \mathrm{~cm}^{3}$ of aqueous dye solution of varying concentrations $(0.03$ to $2.1 \mathrm{mmol} / \mathrm{l})$ with $0.2 \mathrm{~g}$ of the adsorbent in a $250 \mathrm{~cm}^{3}$ conical flask with stopper for three hours. For kinetic studies, $100 \mathrm{~cm}^{3}$ of dye solution at $(0.14 \mathrm{mmol} / \mathrm{l})$ was contacted with $0.2 \mathrm{~g}$ of the adsorbent in a $250 \mathrm{~cm}^{3}$ conical flask with stopper. Samples were taken out periodically and the change in concentration of the dye with time was determined using a UV-Vis spectrophotometer (Spectroscan 2600 from Chemito, India). The amount adsorbed per unit weight of the adsorbent $\left(Q_{t}, \mathrm{mmol} / \mathrm{g}\right)$ at time $t(\mathrm{~min})$ was estimated from the values of initial dye concentration $\left(C_{0}, \mathrm{mmol}\right)$, the dye concentration $\left(C_{t}, \mathrm{mmol}\right)$ at time " $t$ ", the adsorbate volume $\left(V, \mathrm{~cm}^{3}\right)$ and the adsorbent weight $(W, g)$ by the following equation:

$$
Q_{t}(\mathrm{mmol} / \mathrm{g})=\frac{\left(C_{0}-C_{t}\right) \times V}{w \times 1000}
$$

\subsection{Adsorption Isotherm \& Kinetic Equations}

The isotherm data were fitted to Langmuir [16], Freundlich [17] and Temkin [18] adsorption isotherm models. Equations for the Langmuir, Freundlich and Temkin isotherm model are presented as Equations (2)-(4) respectively.

$$
\begin{gathered}
Q_{e}=\frac{b V_{m} C_{e}}{1+b C_{e}} \\
Q_{e}=k_{F} c^{1 / n} \\
Q_{e}=\frac{R T}{b_{T}} \ln A+\frac{R T}{b_{T}} \ln C_{e} \text { and } B=\frac{R T}{b_{T}}
\end{gathered}
$$

where,

$Q_{e}$ : The equilibrium adsorption capacity (mmol/g).

$C_{e}$ : Equilibrium adsorbate (dye) concentration (mmol/l).

$b$ : Langmuir isotherm constant for adsorption bond energy $(\mathrm{l} / \mathrm{mmol})$. 
$V_{m}$ : Langmuir isotherm constant for monolayer adsorption capacity (mmol/g).

$n$ : Freundlich isotherm constant representing adsorption affinity.

$k_{F}$ : Freundlich isotherm constant representing adsorption capacity (mmol/g).

A: Temkin isotherm equilibrium binding constant $(\mathrm{l} / \mathrm{mmol})$.

$b_{T}$ : Temkin isotherm constant.

$R$ : Universal gas constant $(8.314 \mathrm{~J} / \mathrm{mol} / \mathrm{K})$.

$T$ : Absolute temperature (K).

$B$ : Temkin isotherm constant related to heat of sorption $(\mathrm{J} / \mathrm{mmol})$.

The kinetic data were fitted to the standard first order kinetic expression and the Lagergren first order expression [19] as presented in Equations (5) and (6) respectively:

$$
\begin{gathered}
\ln C_{t}=\ln C_{0}-k_{1} t \\
\ln \left(Q_{e}-Q_{t}\right)=\ln Q_{e}-k_{L} t
\end{gathered}
$$

where, $Q_{e}, Q_{t}, C_{0}$ and $C_{t}$ retain their conventional meaning.

$k_{1}$ : Standard first order rate constant.

$k_{L}$ : Lagergren first order rate constant.

\section{Results and Discussions}

\subsection{Characterization of Adsorbents}

Figure 1 shows the X-ray diffraction patterns of the $\mathrm{MgAl}$ and MgAl-Alg composites. The XRD peaks observed for MgAl and its composites having d values $0.757,0.382,0.259,0.229,0.194$ and $0.152 \mathrm{~nm}$ are typical of hydrotalcite-like layered clay structure with characteristic diffraction planes 003, 006, 009, 015, 018 and 110 respectively. The XRD pattern of MgAl-Alg (20\%)

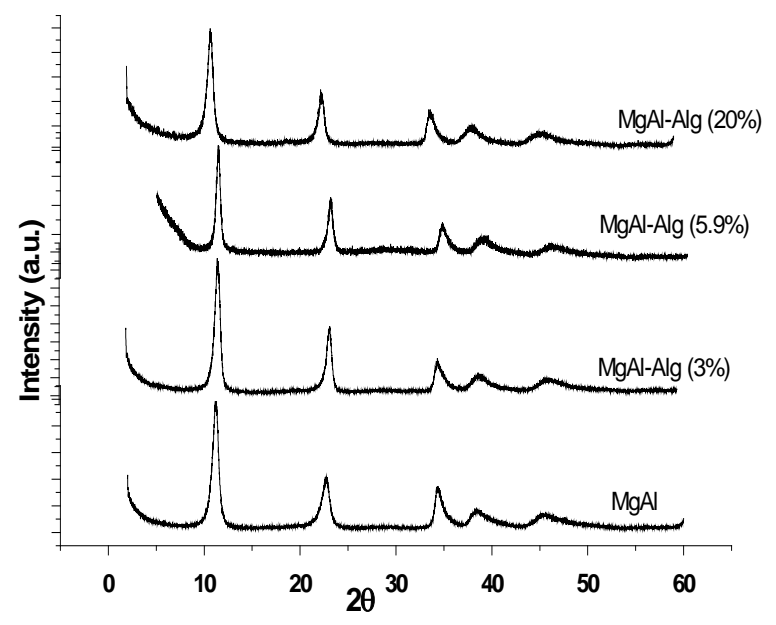

Figure 1. X-ray diffraction patterns of MgAl LDH and MgAl-Alg composites with varying amount of sodium alginate. shows shift in 003 and 006 diffraction peaks, from higher to lower $2 \theta$ values indicating an increase in interlayer space of the layered clay after composite formation. This reveals that alginate ions, when present in high concentration, get intercalated into the interlayer space of the layered clay structure.

Figure 2 shows the SEM images of the pristine clay and its composites. Pristine MgAl crystals are flake-like with sharp edges, stacked in the form of layers (Figure 2(a)) whereas sodium alginate (Figure 2(b)) has a smooth surface. Small alginate particles can be seen spread over the clay surface and in-between layers of the clay, at low alginate concentrations (Figures 2(c) and (d)). At higher concentrations (Figures 2(e) and (f)), alginate starts forming a smooth continuous/semi-continuous layer on the clay.

The specific surface area of MgAl, MgAl-Alg (3\%), MgAl-Alg (5.9\%), MgAl-Alg (11\%) and MgAl-Alg (20\%), determined by nitrogen adsorption-desorption technique, are $51.8,53.7,59.5,52.0$ and $40.0 \mathrm{~m}^{2} / \mathrm{g}$ respectively. The surface area of sodium alginate from nitrogen adsorption-desorption experiments is zero indicating that its surface does not adsorb nitrogen. The surface area of the pristine clay increased on composite formation and increases on increase in alginate concentration up to $5.9 \%$ beyond which it starts decreasing.

\subsection{Influence of Alginate Concentration on Dye Adsorption}

Adsorption capacity of the composites containing different concentration of sodium alginate was evaluated for the two dyes, AG 25 and AG 27. Figure 3 shows that the adsorption capacity for both the dyes vary with sodium alginate concentration and passes through a maximum at an alginate concentration of 5.9\%. The increase in adsorption capacity may be directly correlated with the increase in specific surface area of the composites up to an alginate concentration of $5.9 \%$. Nevertheless, as the adsorption capacities of the composites are higher than the pristine clay ( $0 \%$ sodium alginate) a detailed study has been performed to understand the adsorption mechanism of the layered clay-alginate composites.

\subsection{Adsorption Isotherm}

The adsorption behavior of pristine $\mathrm{MgAl}$ and MgAl-Alg composites with different sodium alginate concentrations have been studied in the initial concentration range of 0.3 - $1.5 \mathrm{mmol} / \mathrm{l}$ for AG 25 and 0.3 - $2.1 \mathrm{mmol} / \mathrm{l}$ for AG 27. Pure sodium alginate exhibited negligible adsorption capacity for the dyes. The adsorption equilibrium data were fitted to three different isotherm models, viz. Langmuir, Freundlich and Temkin. The values of isotherm constants were calculated from the slopes and in- 


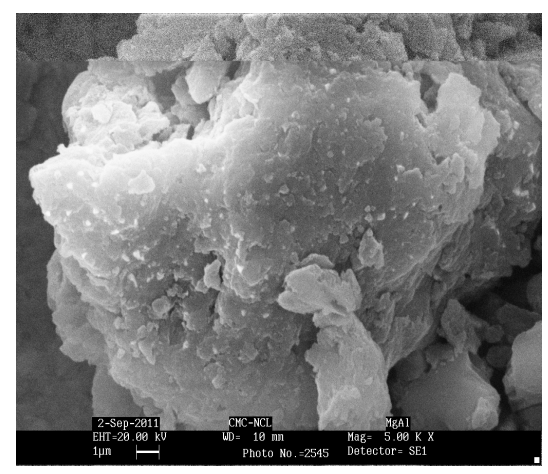

(a)

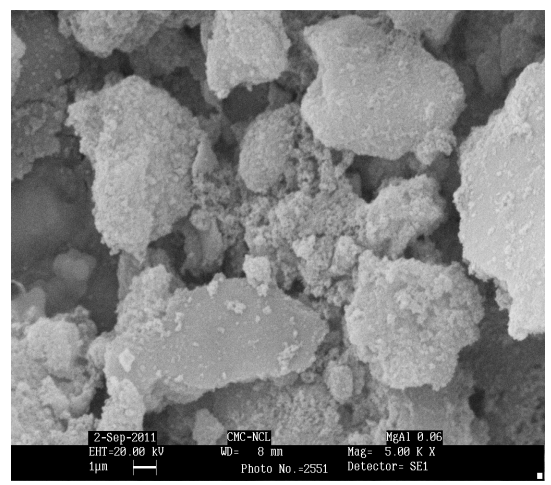

(d)

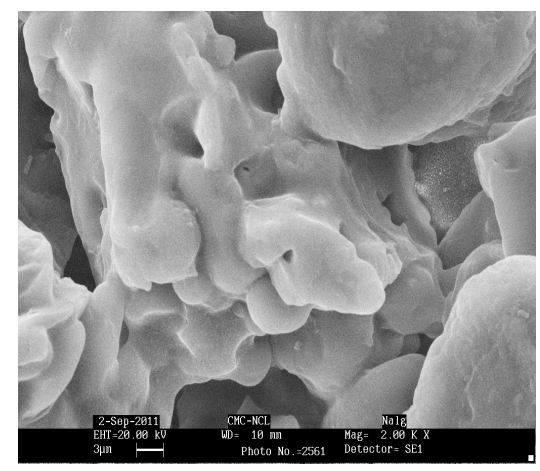

(b)

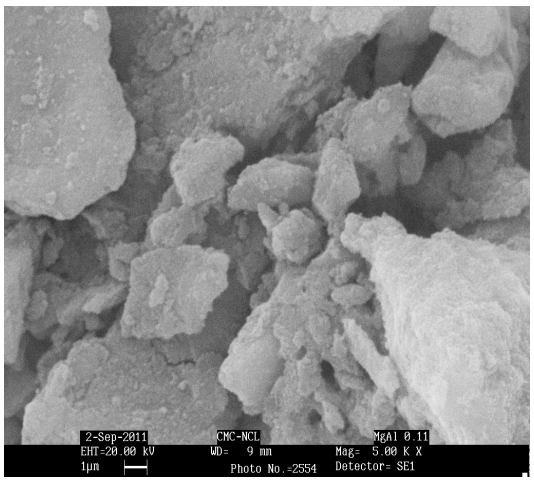

(e)

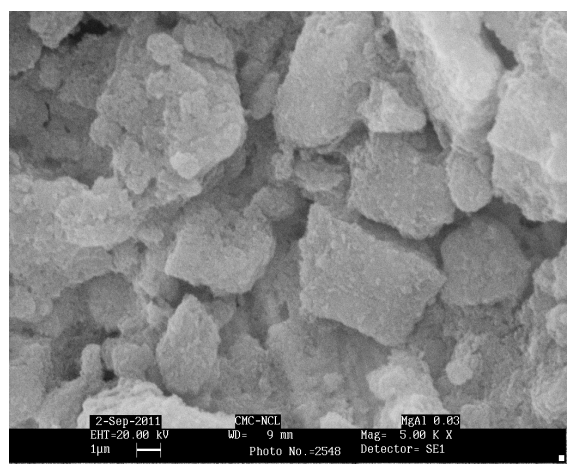

(c)

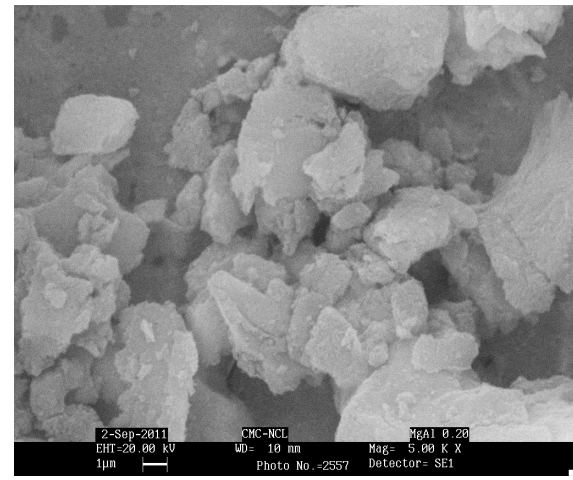

(f)

Figure 2. SEM images of MgAl, Sodium alginate and their composites. (a) MgAl LDH; (b) Sodium alginate; (c) MgAl-Alg (3\%); (d) MgAl-Alg (5.9\%); (e) MgAl-Alg (11\%) and (f) MgAl-Alg (20\%).

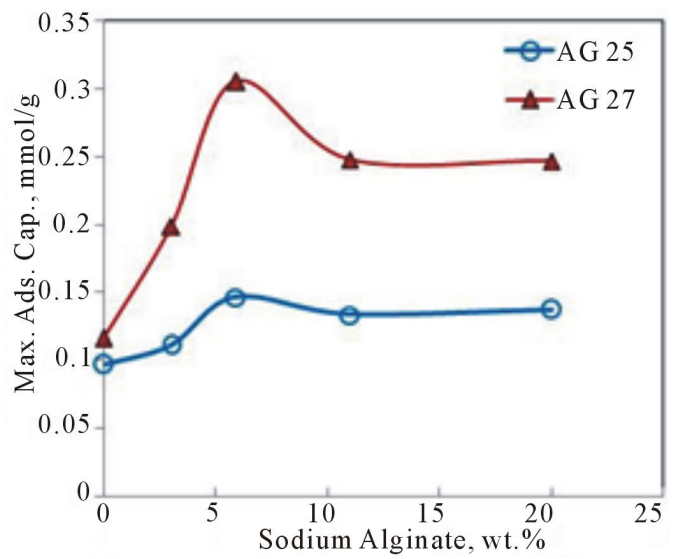

Figure 3. Influence of sodium alginate concentration on the adsorption capacity of MgAl-Alg composites for the dyes, AG 25 and AG 27 (wt. of adsorbent: $0.1 \mathrm{~g}$, vol. of adsorbate: $50 \mathrm{~cm}^{3}$, adsorption time: $3 \mathrm{~h}$, adsorption temp.: $25^{\circ} \mathrm{C}$ ).

tercepts of the linearized plot of the corresponding isotherm equations (Equations (2)-(4)). Table 1 presents the values of different isotherm constants along with the structure and molecular weight of the two dyes.

Table 1 shows that the fitting of experimental data was best ( $R^{2}$ values very close to unity) with Langmuir model for AG 25 and with Freundlich model for AG 27. The values of Langmuir monolayer capacity $\left(V_{m}\right)$ matches well with the Freundlich adsorption constant $\left(k_{F}\right)$ for the dye AG 25. However, for the dye AG 27, the values of Langmuir monolayer capacity $\left(V_{m}\right)$ were little higher than the Freundlich adsorption constant $\left(k_{F}\right)$. The monolayer adsorption capacity of the composite increased up to $50 \%$ for AG 25 and $150 \%$ for AG 27 . For both the dyes, the maximum capacity was achieved by the composite with $5.9 \%$ sodium alginate. The high values of adsorption bond energy (b) show good adsorption characteristics of both the dyes on the composites. The values of affinity constant, n, $(>1)$ of Freundlich model indicate favorable adsorption characteristics of the composites for the dyes [20]. The reasonably low values of Temkin isotherm constant B, related to heat of adsorption, indicates purely physical nature of this adsorption process [20].

The standard adsorption isotherms [equilibrium adsorption capacity Vs equilibrium adsorbate concentration, $Q_{e}$ Vs $C_{e}$ ] for the two dyes AG 25 and AG 27 have been presented in Figure 4 (represented by data points). Figure 4 shows that the standard adsorption isotherms for both dyes represent " $\mathrm{H}$ "-type curve where, $\mathrm{H}$ stands for "high affinity”, a special case of Langmuir isotherm, as proposed by Giles et al. [21]. H-type isotherms are observed in cases when the solute has such high affinity that in dilute solutions it is completely adsorbed. Therefore 
Table 1. Isotherm constants for the adsorption of AG 25 and AG 27 on MgAl and its composites.

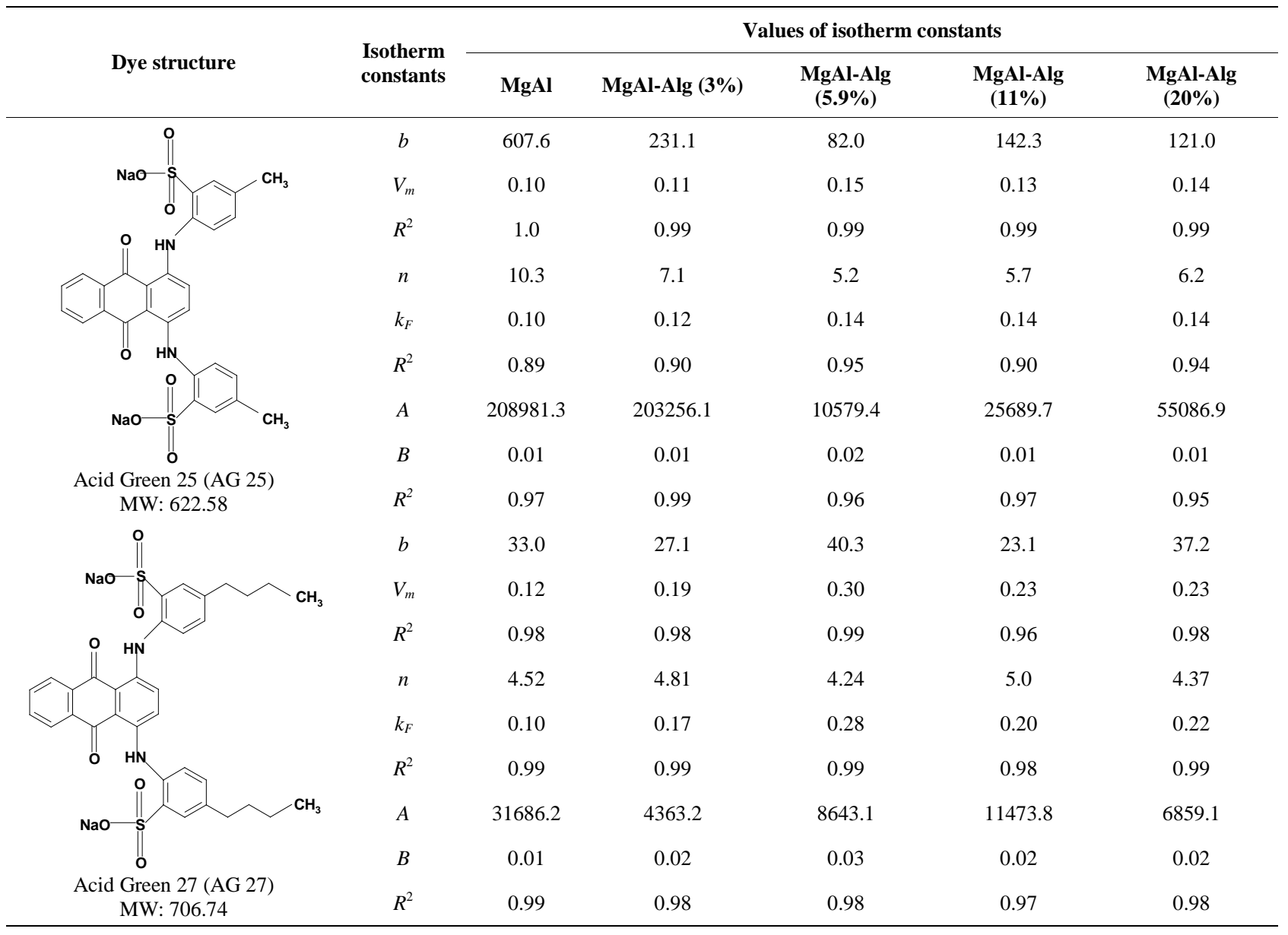

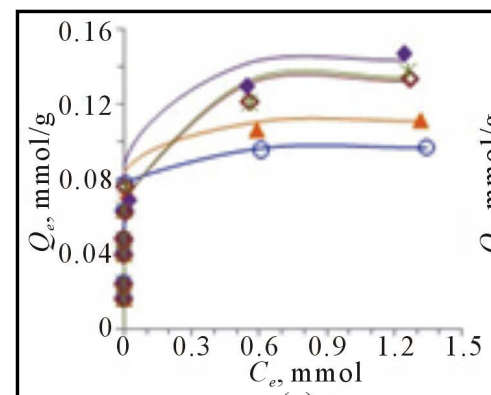

(a)

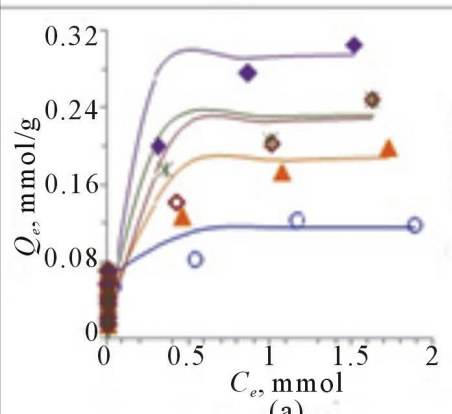

(a)

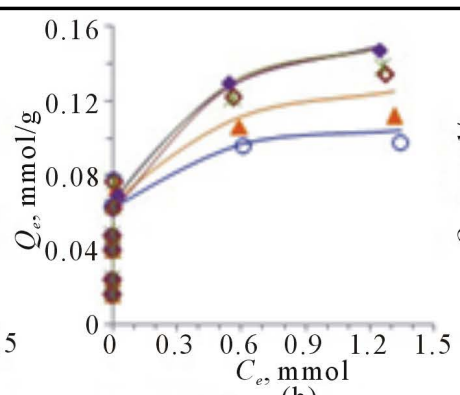

(b)
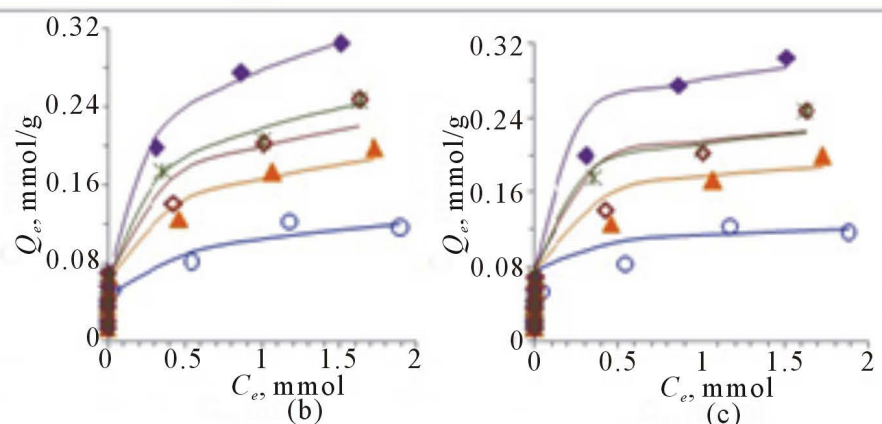

Acid Green 27

- $\mathrm{MgAl}$

4 MgAl-Alg $(3 \%)$

- MgAl-Alg (5.9\%)

$\bullet \mathrm{MgAl}-\mathrm{Alg}(11 \%)$

* MgAl-Alg (20\%)

Figure 4. Adsorption isotherms for AG 25 and AG 27 on MgAl and MgAl-Alg composites (a) Langmuir model; (b) Freundlich model and (c) Temkin model. The data points represent experimental values whereas the lines represent the model fittings. 
the initial part of the isotherm is vertical. Beyond the inflection the curve is of normal L-type (Langmuir-type) isotherm. This indicates the exchange of sulphonated dye ions with chloride ions of the layered clay during the sorption process in a similar fashion as Giles et al. described the exchange of sulphonated dye ions with chloride ions on alumina surface [21].

The equilibrium adsorption capacity $\left(Q_{e}\right)$ generated using different isotherm models are presented as continuous line in Figure 4. Although the Langmuir isotherm model gave an excellent fitting to the experimental adsorption data in the initial region, it did not match well with the experimental data points at higher adsorbate concentration. In contrast, $Q_{e}$ values generated using Freundlich isotherm model show excellent match with the experimental data indicating heterogeneity in the adsorbent sites for the adsorption of AG 25 and AG 27.

\subsection{Adsorption Kinetics}

The kinetic data for the adsorption of the dyes AG 25 and AG 27 on $\mathrm{MgAl}$ and its composites with sodium alginate was fitted to the standard first order equation as well as the Lagergren models. The rate constants based on these models and the respective regression coefficients are presented in Table 2. Table 2 shows that the kinetic data can be presented well by both the first order rate equation and the Lagergren equation.

The values of kinetic constants $k_{1}$ and $k_{L}$ are higher in case of the composites as compared to the pristine LDH. Opening of the interlayer spaces of the LDH by the alginate ions/particles may increase the accessibility of the interlayer sites, in the case of adsorption on the composite. This decrease in resistance can lead to the observed high values of the rate constant in the case of MgAl-Alg composites. The concentration and the amounts adsorbed were predicted for different time intervals using the standard first order and Lagergren rate equations respectively for the adsorption of AG 25 and AG 27 on MgAl and MgAl-Alg composites with alginate concentration of $3 \%, 5.9 \%, 11 \%$ and $20 \%$. The predicted values for AG 25 and AG 27 are plotted against experimental ones and presented in Figures 5(a) and (b). The diagonal represents

Table 2. Kinetic constants and the regression coefficients for adsorption of AG 25 and AG 27 on MgAl and MgAl-Alg composites.

\begin{tabular}{|c|c|c|c|c|c|c|}
\hline \multirow{2}{*}{ Adsorbate } & \multirow{2}{*}{ Constants } & \multicolumn{5}{|c|}{ Alginate concentration (wt\%) } \\
\hline & & $\mathbf{0}$ & 3 & 5.9 & 11 & 20 \\
\hline \multirow{4}{*}{ AG 25} & $\mathrm{k}_{1}$ & 0.026 & 0.020 & 0.036 & 0.027 & 0.023 \\
\hline & $\mathrm{R}^{2}$ & 0.921 & 0.876 & 0.787 & 0.822 & 0.676 \\
\hline & $\mathrm{k}_{\mathrm{L}}$ & 0.031 & 0.026 & 0.046 & 0.038 & 0.042 \\
\hline & $\mathrm{R}^{2}$ & 0.928 & 0.901 & 0.939 & 0.931 & 0.877 \\
\hline \multirow{4}{*}{ AG 27} & $\mathrm{k}_{1}$ & 0.013 & 0.022 & 0.043 & 0.034 & 0.037 \\
\hline & $\mathrm{R}^{2}$ & 0.898 & 0.808 & 0.712 & 0.835 & 0.784 \\
\hline & $\mathrm{k}_{\mathrm{L}}$ & 0.027 & 0.035 & 0.065 & 0.049 & 0.055 \\
\hline & $\mathrm{R}^{2}$ & 0.935 & 0.894 & 0.794 & 0.956 & 0.867 \\
\hline
\end{tabular}
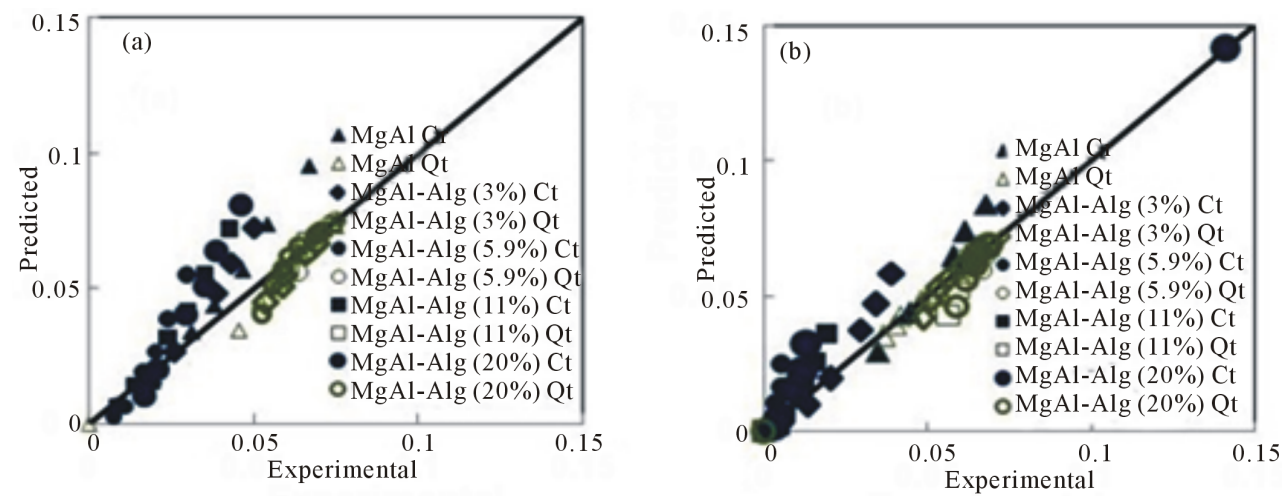

Figure 5. Parity plots for the first order and Lagergren models for the kinetics of adsorption of the dyes (a) AG 25 and (b) AG 27 on MgAl and its composites containing different alginate concentrations. Solid points represent $C_{t}$ values, experimental versus predicted by first order rate equation; hollow points represent $Q_{t}$ values, experimental versus those predicted by the Lagergren model. 
the trend when the prediction matches with the experimental values. The solid points in both the figures represent the conventional first order kinetic equation whereas the hollow points represent the Lagergren kinetic model. Figures 5(a) and (b) show that the $C_{t}$ values are over predicted by the first order equation. The $Q_{t}$ values obtained using the Lagergren model do not show much scatter indicating that this model is a better representation of the adsorption kinetics. This is also supported by the value of correlation coefficients in Table 2 . In the case of both the models, the rate constants $k_{1}$ and $k_{L}$ exhibit higher value for the composite compared to the pristine $\mathrm{LDH}$. The value is the highest for an alginate concentration of $5.9 \%$.

\subsection{Adsorption Mechanism}

The adsorbates AG 25 and AG 27 are anionic dyes. The mechanism of adsorption of these dyes on the anionic clay and its composites is expected to be adsorption and ion-exchange. AG 25 and AG 27 are bulky anthraquinone based dyes and there can be resistance to their penetration into the interlayer spaces of the anionic clay. Hence it is expected that these dyes will be interacting with the external surface of adsorbate by both ion-exchange and physisorption, with equal ease. AG 25 and AG 27 are similar except for the fact that the $-\mathrm{CH}_{3}$ groups in $\mathrm{AG} 25$ is replaced by the longer $-\mathrm{C}_{4} \mathrm{H}_{9}$ groups in AG 27. These butyl groups present in AG 27 may increase the penetration of this dye into the interlayer spaces of the LDH and LDH composites. The bond energy for the adsorption, the constant $b$ in the Langmuir Isotherm (Table 1) is higher for the adsorption of AG 25 on the clay studied and the clay-alginate composites. The high value observed for AG 25 may be a reflection of the adsorption of this dye, predominantly by anion-exchange, occurring on the absorbent surface. The variation of Lagergren rate constants as a function of alginate concentration is presented in Figure 6. The figure shows that the adsorption rate constant for AG 27 is higher compared to AG 25. As discussed earlier, the butyl groups in AG 27 might be easing the penetration of the dye into the interlayer spaces of the adsorbent. Hence the rate constant $k_{L}$ for the adsorption of AG 27 is higher compared to AG 25. Figure 6 reveals that the rate of adsorption of both AG 25 and AG 27 are sensitive to the sodium alginate concentration. The rate is maximum at an alginate concentration of $5.9 \%$. At this alginate concentration, the rate is increased by $48 \%$ for AG 25 and by $141 \%$ for AG 27 . This increase in rate may be due to the opening up of the interlayer spaces of MgAl by the alginate. As the alginate concentration further increases, it being a polymer, forms smooth continuous patches on the clay surface which may be hindering the adsorption of the mole-

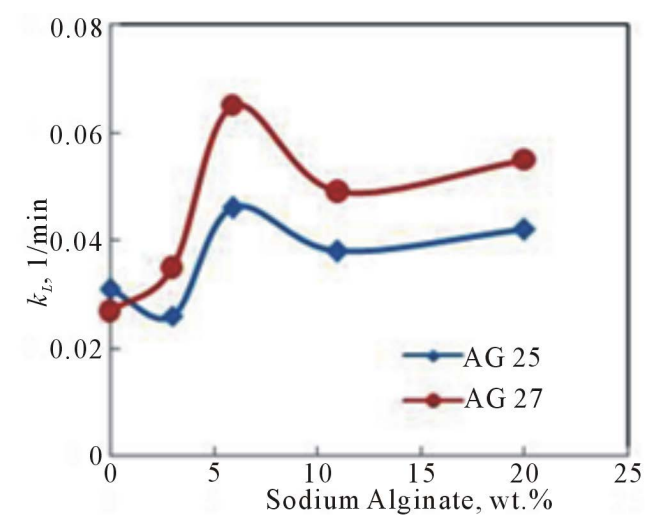

Figure 6. Influence of sodium alginate concentration on the on the Lagergren rate constant of MgAl-Alg composites for AG 25 and AG 27 (wt. of adsorbent: 0.2 g, vol. of adsorbate: $100 \mathrm{~cm}^{3}$, adsorption time: $2 \mathrm{~h}$, adsorption temperature: $\left.25^{\circ} \mathrm{C}\right)$.

cules. This also leads to a decrease in the adsorption capacity of the adsorbents as can be seen in Figure 3 .

\section{Conclusion}

The anionic clay MgAl and its composites with sodium alginate, a biocompatible natural polymer, were synthesized and characterized. The adsorption properties of these materials were studied for the adsorption of anthraquinone dyes, AG 25 and AG 27. The adsorption behaviour of the composites was superior to that of the pristine clay, however, varied with the alginate concentration in the composite. The adsorption rate and capacity of the dyes on the composites followed the order AG $27>$ AG 25. Comparison of the adsorption characteristics of pristine clay reveals that the maximum adsorption capacity of the bio-composite increase by $51 \%$ and $160 \%$ in the case of adsorbates AG 25 and AG 27, respectively. The maximum adsorption rate increases by $48 \%$ and 147\% for the adsorbates AG 25 and AG 27, respectively. This has been discussed in terms of the structure of the dye and the surface changes of pristine clay on composite formation. Among the composites, the one with alginate concentration of $5.9 \%$ had the highest adsorption capacity for all the dyes studied. The results reveal that these composites may be used for removal of these dyes from aqueous effluents.

\section{REFERENCES}

[1] F, Gao, “Clay-Polymer Composites: The Story,” Materials Today, Vol. 7, No. 11, 2004, pp. 50-55. http://dx.doi.org/10.1016/S1369-7021(04)00509-7

[2] R. Srinivasan, "Advances in Application of Natural Clay and Its Composites in Removal of Biological, Organic, and Inorganic Contaminants from Drinking Water," Advances in Materials Science and Engineering, Vol. 2011, 2011, Article ID: 872531. 
[3] F. D. Alsewailem and S. A. Aljlil, "Recycled Polymer/ Clay Composites for Heavy-Metals Adsorption,” Materials Technology, Vol. 47, 2013, pp. 525-529.

[4] D. S. Dlamini, A. K. Mishra and B. B. Mamba, "Morphological, Transport, and Adsorption Properties of Ethylene Vinyl Acetate/Polyurethane/Bentonite Clay Composites,” Journal of Applied Polymer Science, Vol. 124, 2012, pp. 4978-4985.

[5] M. Auta and B. H. Hameed, "Chitosan-Clay Composite as Highly Effective and Low-Cost Adsorbent for Batch and Fixed-Bed Adsorption of Methylene Blue,” Chemical Engineering Journal, Vol. 237, 2014, pp. 352-361. http://dx.doi.org/10.1016/j.cej.2013.09.066

[6] N. Bleiman and Y. G. Mishael, "Selenium Removal from Drinking Water by Adsorption to Chitosan-Clay Composites and Oxides: Batch and Columns Tests,” Journal of Hazardous Materials, Vol. 183, No. 1-3, 2010, pp. 590595. http://dx.doi.org/10.1016/j.jhazmat.2010.07.065

[7] R. Ganigar, G. Rytwo, Y. Gonen, A. Radian and Y. G. Mishael, "Polymer-Clay Nanocomposites for the Removal of Trichlorophenol and Trinitrophenol from Water," Applied Clay Science, Vol. 49, No. 3, 2010, pp. 311-316. http://dx.doi.org/10.1016/j.clay.2010.06.015

[8] D. S. Dlamini, A. K. Mishra and B. B. Mamba, “ANN Modeling in $\mathrm{Pb}$ (II) Removal from Water by Clay-Polymer Composites Fabricated via the Melt-Blending," Journal of Applied Polymer Science, Vol. 130, 2013, pp. 38943901.

[9] N. Viswanathan and S. Meenakshi, "Selective Fluoride Adsorption by a Hydrotalcite/Chitosan Composite," Applied Clay Science, Vol. 48, No. 4, 2010, pp. 607-611. http://dx.doi.org/10.1016/j.clay.2010.03.012

[10] X. Ruan, S. Huang, H. Chen and G. Qian, "Sorption of Aqueous Organic Contaminants onto Dodecyl Sulfate Intercalated Magnesium Iron Layered Double Hydroxide,” Applied Clay Science, Vol. 72, 2013, pp. 96-103. http://dx.doi.org/10.1016/j.clay.2013.01.001

[11] S. Mandal and S. Mayadevi, "Cellulose Supported Layered Double Hydroxides for the Adsorption of Fluoride from Aqueous Solution,” Chemosphere, Vol. 72, No. 6, 2008, pp. 995-998. http://dx.doi.org/10.1016/j.chemosphere.2008.03.053

[12] S. Mandal and S. Mayadevi, "Defluoridation of Water Using As-Synthesized Zn/Al/Cl Anionic Clay Adsorbent:
Equilibrium and Regeneration Studies,” Journal of Hazardous Materials, Vol. 167, No. 1-3, 2009, pp. 873-878. http://dx.doi.org/10.1016/j.jhazmat.2009.01.069

[13] F. Bruna, R. Celis, I. Pavlovic, C. Barriga, J. Cornejo and M. A. Ulibarri, "Layered Double Hydroxides as Adsorbents and Carriers of the Herbicide (4-Chloro-2-Methylphenoxy) Acetic Acid (MCPA): Systems Mg-Al, Mg-Fe and Mg-Al-Fe," Journal of Hazardous Materials, Vol. 168, No. 2-3, 2009, pp.1476-1481. http://dx.doi.org/10.1016/j.jhazmat.2009.03.038

[14] Z. Zhou, G. Y. Li and Y. J. Li, "Immobilization of Saccharomyces Cerevisiae Alcohol Dehydrogenase on Hybrid Alginate-Chitosan Beads," International Journal of Biological Macromolecules, Vol. 47, No. 1, 2010, pp. 2126. http://dx.doi.org/10.1016/j.ijbiomac.2010.04.001

[15] S. Mandal, V. S. Patil and S. Mayadevi, “Alginate and Hydrotalcite-Like Anionic Clay Composite Systems: Synthesis, Characterization and Application Studies," Microporous and Mesoporous Materials, Vol. 158, 2012, pp. 241-246. http://dx.doi.org/10.1016/j.micromeso.2012.03.046

[16] I. Langmuir, "The Adsorption of Gases in Plane Surface of Glass, Mica and Platinum," Journal of American Chemical Society, Vol. 40, No. 9, 1916, pp. 1361-1368. http://dx.doi.org/10.1021/ja02242a004

[17] H. M. F. Freundlich, "Over the Adsorption in Solution," Journal of Physical Chemistry, Vol. 57, 1906, pp. 385470.

[18] C. Aharoni and D. L. Sparks, "Kinetics of Soil Chemical Reaction: A Theoretical Treatment, Soil Science Society of America," In: D. L. Sparks and D. L. Suarez, Eds., Madison, 1991, pp. 1-18.

[19] S. Lagergren, "About the Theory of So-Called Adsorption of Soluble Substance,” Handlingar, Vol. 24, 1898, pp. 139.

[20] A. O. Dada, A. P. Olalekan, A. M. Olatunya and O. Dada, "Langmuir, Freundlich, Temkin and Dubinin-Radushkevich Isotherms Studies of Equilibrium Sorption of $\mathrm{Zn}^{2+}$ onto Phosphoric Acid Modified Rice Husk,” IOSR Journal of Applied Chemistry, Vol. 3, No. 1, 2012, pp. 38-45.

[21] C. H. Giles, T. H. MacEwan, S. N. Nakhwa and D. Smith, "Studies in Adsorption," Journal of Chemical Society, 1960, pp. 3973-3993. 